\title{
Actinin-4 protein overexpression as a predictive biomarker in adjuvant chemotherapy for resected lung adenocarcinoma
}

\author{
Hideaki Shiraishi',2, Yutaka Fujiwara ${ }^{1,3}$, Takanori Kakuya ${ }^{4}$, Koji Tsuta ${ }^{5}$, Noriko Motoi ${ }^{5}$, \\ Nami Miura ${ }^{4}$, Yukio Watabe ${ }^{4}$, Shun-ichi Watanabe ${ }^{6}$, Rintaro Noro ${ }^{4}$, Kengo Nagashima ${ }^{7}$, \\ Wilber Huang ${ }^{8}$, Tesshi Yamada ${ }^{4}$, Hisao Asamura ${ }^{9}$, Yuichiro Ohe ${ }^{1,2}$ \& Kazufumi Honda*,4,10 \\ ${ }^{1}$ Department of Thoracic Oncology, National Cancer Center Hospital, Tokyo, Japan \\ ${ }^{2}$ Department of Course of Advanced Clinical Research of Cancer, Juntendo University Graduate School of Medicine, Tokyo, Japan \\ ${ }^{3}$ Department of Experimental Therapeutics, Exploratory Oncology Research \& Clinical Trial Center, National Cancer Center \\ Hospital, Tokyo, Japan \\ ${ }^{4}$ Division of Chemotherapy \& Clinical Research, National Cancer Center Research Institute, Tokyo, Japan \\ ${ }^{5}$ Pathology \& Clinical Laboratory Division, National Cancer Center Hospital, Tokyo, Japan \\ ${ }^{6}$ Department of Thoracic Surgery, National Cancer Center Hospital, Tokyo, Japan \\ ${ }^{7}$ Department of Global Clinical Research, Graduate School of Medicine, Chiba University, Chiba, Japan \\ ${ }^{8}$ Abnova Corporation, Taipei, Taiwan \\ ${ }^{9}$ General Thoracic Surgery, School of Medicine, Keio University, Tokyo, Japan \\ ${ }^{10}$ Japan Agency for Medical Research \& Development: AMED-CREST, AMED, Tokyo, Japan \\ * Author for correspondence: khonda@ncc.go.jp
}

\begin{abstract}
Aim: Although several clinical trials demonstrated the benefits of platinum-combination adjuvant chemotherapy for stage II-IIIA lung adenocarcinoma, predictive biomarkers for the efficacy of such therapy have not yet been identified. We evaluated protein overexpression of actinin- 4 as a predictive biomarker of the efficacy of adjuvant chemotherapy in resected lung adenocarcinoma. Materials \& methods: We measured actinin-4 protein levels in patients with completely resected stage II-IIIA lung adenocarcinoma using immunohistochemistry and then retrospectively compared survival between adjuvant chemotherapy and observation groups. Results: A total of 148 eligible patients were classified into actinin-4 positive or negative cases by immunohistochemistry. In the former, patients with adjuvant chemotherapy survived significantly longer than those with observation (hazard ratio $[H R]: 0.307 ; p=0.028$ ). But, no significant survival benefit was noted with adjuvant chemotherapy (HR: $0.926 ; p=0.876$ ) in the latter. Conclusion: This marker could predict the efficacy of adjuvant chemotherapy for resected lung adenocarcinoma patients.
\end{abstract}

First draft submitted: 1 May 2017; Accepted for publication: 13 June 2017; Published online: 29 June 2017

Keywords: actinin-4 (ACTN4) • adjuvant chemotherapy • predictive biomarker

Non-small-cell lung cancer (NSCLC) is one of the most lethal malignancies worldwide. Surgical resection is the most potentially curative treatment and is the standard treatment choice for patients with early stage NSCLC. However, despite curative surgery, the 5-year survival of NSCLC patients is $46-64 \%$ at stage IIA, about $36-49 \%$ at stage IIB and just $25 \%$ at stage IIIA [1,2]. Several meta-analyses and randomized controlled trials, including the JBR. 10 and ANITA trials, have demonstrated the efficacy of adjuvant chemotherapy for NSCLC patients [3-6]. Although adjuvant cisplatin-based chemotherapy is recommended as standard care in completely resected NSCLC patients with stage II-IIIA $[7,8]$, the relative reduction in the risk of death of patients undergoing adjuvant chemotherapy is modest in comparison with that of patients without adjuvant chemotherapy (hazard ratio [HR] for stage II: 0.83; 95\% CI: 0.73-0.95; and HR for stage III: 0.83; 95\% CI: 0.72-0.94). Therefore, the identification of predictive biomarkers for the efficacy of adjuvant chemotherapy for stage II-IIIA is required in the clinical setting. To identify companion diagnostic biomarkers for adjuvant chemotherapy, numerous prognostic factors as well as predictive factors for adjuvant chemotherapy have been investigated for early stage NSCLC [9-14]. 
Actinin-4 (gene name: ACTN4) is an actin-bundling protein that directly regulates cell motility through remodeling of the actin cytoskeleton [15]. We recently developed a practical method for the detection of actinin-4 protein expression using immunohistochemistry (IHC). The use of this method showed that actinin- 4 protein expression is closely associated with poor outcomes in patients with various cancers [15-18]. Overexpression of actinin-4 induces the formation of cellular processes termed filopodia and lamellipodia that are closely associated with cell motility and cancer invasion, and confer a highly metastatic phenotype on cancer cells [19]. It is thought that adjuvant chemotherapy controls latent metastatic lesions that could not have been detected by imaging modalities. If there were a biomarker that could evaluate the metastatic ability of cancer cells, it may be possible to predict the efficacy of adjuvant chemotherapy.

A retrospective study was performed to assess the efficacy and feasibility of adjuvant platinum-combination chemotherapy for patients with stage II-IIIA lung adenocarcinoma, as well as the utility of actinin- 4 as a predictive biomarker for such patients.

\section{Materials \& methods}

Study population

A total of 1076 patients with adenocarcinoma of the lung underwent complete surgical resection at the National Cancer Center Hospital in Japan from January 2008 to December 2011. Eligible subjects were those with a histologically confirmed lung adenocarcinoma with pathological stage IIA, IIB or IIIA in accordance with tumor node metastasis staging (seventh edition) [20] and with adjuvant platinum-combination chemotherapy. Ineligible subjects are described in Figure 1. Ultimately, 148 patients with adenocarcinoma of the lung were enrolled according to the Reporting Recommendations for Tumor Marker Prognostic Studies (REMARK) [21] (Figure 1). This study was conducted with the approval of the Institutional Review Board of the National Cancer Center. The procedures followed were in accordance with the ethical standards of the responsible committee on human experimentation (institutional or regional) and with the Helsinki Declaration of the World Medical Association.

\section{Patient data}

The following data were extracted: patient characteristics: age, sex and Eastern Cooperative Oncology Group Performance Status (ECOG PS) at the start of adjuvant chemotherapy, smoking status, Charlson Comorbidity Index (CCI), pathological stage after surgery, type of surgery and histological diagnosis before and after surgery; type of chemotherapeutic agents administered, dose, treatment cycle and toxicity;) recurrence patterns assessed by follow-up imaging studies and by more invasive procedures, including bronchoscopy and computed tomographyguided needle biopsies, in cases requiring a pathological diagnosis; and recurrence-free survival (RFS) and overall survival (OS). Local recurrences were defined as those at the surgical margins, the bronchial stump or in the regional lymph nodes. Distant recurrences were defined as those in solid organs, such as residual lung, brain, bone or lymph nodes that were not resected in the primary operation. All patients gave their written, informed consent to analyze their medical records after treatments.

\section{Immunohistochemical staining for actinin-4 \& assessment}

Formalin-fixed paraffin-embedded tissue specimens from resected lung adenocarcinomas were immunohistochemically analyzed with an anti-human actinin- 4 monoclonal antibody (N-terminal actinin-4) (Abnova Corporation, Taipei, Taiwan) [17,22]. Immunoperoxidase staining was performed as described. Immunoreactivity was classified as positive or negative as follows. Positive expression of actinin-4 protein by IHC in cancer cells was defined as a positive reaction that was more intense than that of vascular endothelial cells present in the same section and was evident in more than $30 \%$ of the tumor area. Any lower level of immunoreactivity was classified as IHC negative. The criterion (>30\%) for assessment of ACTN4 expression by IHC was defined according to the previous study in which we evaluated the expression status of ACTN4 in three independent cohorts totaling 1033 patients with stage I lung adenocarcinoma [22]. IHC results were judged independently by three investigators (H Shiraishi, Y Watabe and T Kakuya) who were unaware of the clinical data, and consensus was reached for any discordant cases. The utility of this criterion was proven in our previous studies involving salivary gland carcinoma [17] and pancreatic cancer $[17,22,23]$. 


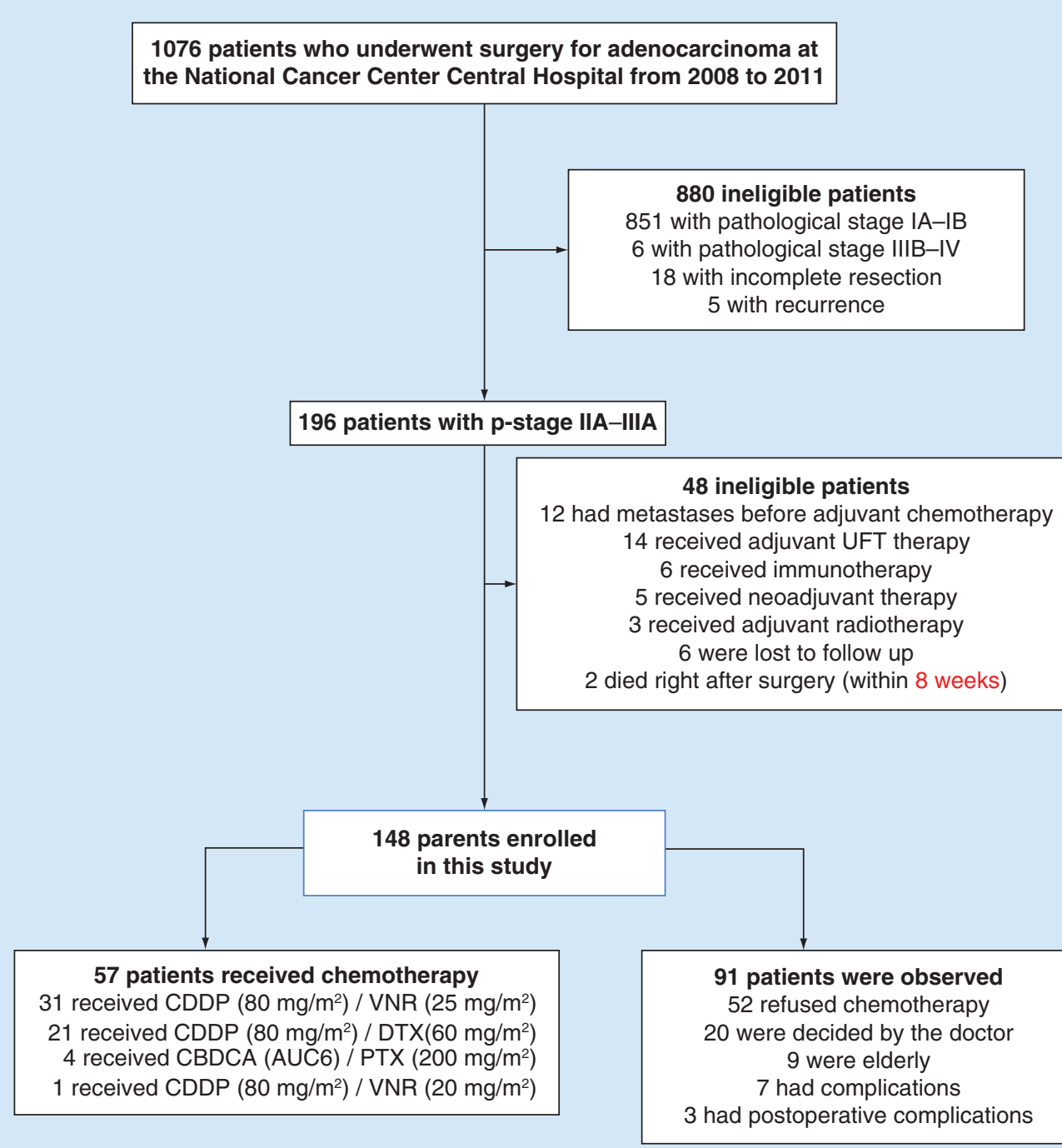

Figure 1. Eligibility of patients with stage II and IIIA adenocarcinoma of the lung. Out of 1076 patients who underwent complete surgery for adenocarcinoma between 2008 and 2011, 148 patients with stage II and IIIA adenocarcinoma of the lung according to Reporting Recommendations for Tumor Marker Prognostic Studies (REMARK) were enrolled.

AUC: Area under the concentration-time curve; CBDCA: Carboplatin; CDDP: Cisplatin; DTX: Docetaxel; PTX: Paclitaxel; VNR: Vinorelbine.

\section{Statistical analysis}

Exploratory analyses were performed to characterize relationships between marker levels, baseline clinical characteristics and survival. Comparisons of baseline characteristics were performed using the $\chi^{2}$ test, Fisher's exact test or the Mann-Whitney test. Kaplan-Meier product-limit methods and the log-rank test were used to estimate and test overall survival distributions and their differences between markers and treatment arms, and multivariable Cox regression models were used to validate the prognostic and predictive effects of markers on survival while adjusting for baseline factors. All reported $\mathrm{p}$-values are two-sided, and a level of $5 \%(\mathrm{p}=0.05)$ was considered significant. These analyses were performed using the SPSS statistical package (SPSS ver. 21.0 for Windows; SPSS Inc., IL, USA). 


\section{Table 1. Baseline patient characteristics.}

\begin{tabular}{|c|c|c|c|}
\hline \multirow[t]{2}{*}{ Factor } & \multicolumn{3}{|c|}{ All patients } \\
\hline & Obs $(n=91)$ & $\operatorname{Adj}(n=57)$ & p-value \\
\hline \multicolumn{4}{|l|}{ Age (years) } \\
\hline Median (range) & $68(49-83)$ & $60(30-75)$ & $<0.01^{\dagger}$ \\
\hline \multicolumn{4}{|l|}{ Sex } \\
\hline Male & $46(51 \%)$ & $30(53 \%)$ & $0.805^{\S}$ \\
\hline Female & $45(49 \%)$ & $27(47 \%)$ & \\
\hline 0 & $51(56 \%)$ & $48(84 \%)$ & $<0.01^{\S}$ \\
\hline 1 & $40(44 \%)$ & $9(16 \%)$ & \\
\hline \multicolumn{4}{|l|}{ Smoking status } \\
\hline Never a smoker & $36(40 \%)$ & $19(33 \%)$ & $0.446^{\S}$ \\
\hline Ex-smoker & $55(60 \%)$ & $38(67 \%)$ & \\
\hline \multicolumn{4}{|l|}{ Tumor size $(\mathrm{cm})$} \\
\hline Median (range) & $3.2(1.1-14.0)$ & $2.5(1.2-7.5)$ & $<0.01^{\dagger}$ \\
\hline \multicolumn{4}{|l|}{ T status } \\
\hline $\mathrm{T} 1$ & $23(25 \%)$ & $30(53 \%)$ & $<0.01^{\ddagger}$ \\
\hline $\mathrm{T} 2$ & $37(41 \%)$ & $16(28 \%)$ & \\
\hline T3 & $26(29 \%)$ & $10(18 \%)$ & \\
\hline T4 & $5(5 \%)$ & $1(1 \%)$ & \\
\hline \multicolumn{4}{|l|}{$\mathrm{N}$ status } \\
\hline No & $25(27 \%)$ & $5(9 \%)$ & $<0.01^{\S}$ \\
\hline N1 & $39(43 \%)$ & $24(42 \%)$ & \\
\hline Bilobectomy & $5(6 \%)$ & $2(3 \%)$ & \\
\hline Pneumonectomy & $1(1 \%)$ & $1(2 \%)$ & \\
\hline Other & $3(3 \%)$ & $1(2 \%)$ & \\
\hline \multicolumn{4}{|l|}{$\begin{array}{l}{ }^{\dagger} \text { Mann-Whitney test. } \\
\ddagger \text { Fisher's exact test. } \\
{ }^{\ddagger} \chi 2 \text { test was used for } \\
\text { Adj: Adjuvant chemot } \\
\text { p-TNM Stage: UICC (U }\end{array}$} \\
\hline
\end{tabular}

\section{Results}

\section{Baseline patient characteristics}

Selection of the 148 patients with stage II or IIIA adenocarcinoma of the lung is summarized in Figure 1. The clinical characteristics of these enrolled patients are shown in Tables 1 and 2. Out of these 148 patients, 91 (61.5\%) were observed without adjuvant therapy (observation [Obs] group), and 57 (38.5\%) underwent adjuvant chemotherapy with platinum-combination therapy (adjuvant chemotherapy (Adj) group) after complete resection of the tumor. Seventy-five patients were actinin-4-IHC-positive $(\mathrm{IHC}(+))$, and 73 were actinin-4-IHC-negative (IHC(-)). Significant differences in baseline characteristics between Obs and Adj group patients were seen for age, ECOG PS, tumor size, T-status, N-status and CCI, and a significant difference between actinin-4-IHC(+) and 
Table 2. Baseline characteristics of the patients with positive/negative immunohistochemical staining for actinin-4.

\begin{tabular}{|c|c|c|c|}
\hline & \multicolumn{2}{|l|}{ All patients } & \multirow[t]{2}{*}{$\mathrm{p}$-value } \\
\hline & IHC+ $(n=75)$ No. $(\%)$ & IHC- $(n=73)$ No. $(\%)$ & \\
\hline \multicolumn{4}{|l|}{ Age (years) } \\
\hline Median (range) & $67(35-83)$ & $65(30-83)$ & $0.115^{\dagger}$ \\
\hline Male & $44(59 \%)$ & $32(44 \%)$ & $0.071^{\ddagger}$ \\
\hline Female & $31(41 \%)$ & $41(56 \%)$ & \\
\hline Ex-smoker & $51(68 \%)$ & $42(58 \%)$ & \\
\hline \multicolumn{4}{|l|}{$\mathrm{p}$-TNM stage } \\
\hline$\| A / B$ & $40(53 \%)$ & $39(56 \%)$ & $0.991^{\ddagger}$ \\
\hline IIIA & $35(47 \%)$ & $34(46 \%)$ & \\
\hline \multicolumn{4}{|l|}{ Tumor size $(\mathrm{cm})$} \\
\hline $\mathrm{T} 3 / 4$ & $25(33 \%)$ & $17(23 \%)$ & \\
\hline \multicolumn{4}{|l|}{$\mathrm{N}$ factor } \\
\hline No & $19(25 \%)$ & $11(15 \%)$ & $0.120^{\ddagger}$ \\
\hline $\mathrm{N} 1 / 2$ & $56(75 \%)$ & $62(85 \%)$ & \\
\hline \multicolumn{4}{|c|}{ Adjuvant chemotherapy } \\
\hline Non (Obs) & $53(71 \%)$ & $38(52 \%)$ & $0.020^{\ddagger}$ \\
\hline Yes (Adj) & $22(29 \%)$ & $35(48 \%)$ & \\
\hline \multicolumn{4}{|l|}{ Type of surgery } \\
\hline Lobectomy & $68(91 \%)$ & $67(92 \%)$ & $0.989^{\ddagger}$ \\
\hline Bilobectomy & $4(5 \%)$ & $3(4 \%)$ & \\
\hline
\end{tabular}

IHC(-) group patients was seen for the presence of adjuvant chemotherapy. The details of adjuvant chemotherapy regimens, drug delivery, toxicity and recurrence patterns are shown in Supplementary Tables 1A \& B and 2A-C.

\section{Prognostic impact of actinin-4 protein expression in the observation group of patients with} adenocarcinoma of the lung

Figure 2A-C shows representative actinin-4 protein expression patterns in pathological sections resected from patients with adenocarcinoma of the lung. Actinin-4 protein expression was not observed in the epithelia of the bronchial tubes and alveolus (Figure 2A). Representative negative and positive staining patterns are shown in Figure 2B \& C, respectively.

We previously reported that actinin-4 protein overexpression is a prognostic biomarker for adenocarcinoma of the lung. This is the first time that the prognostic impact of actinin- 4 protein expression was examined in patients who never underwent adjuvant chemotherapy.

The OS of actinin-4-IHC(+) patients was shorter than that of actinin-4-IHC(-) patients ( $\mathrm{p}=0.021$; log-rank test) (Supplementary Figure 1). Univariate Cox regression analysis for death showed no significant differences associated with age, sex, smoking status, p-stage or CCI. However, the HR of the actinin-4-IHC $(+)$ group was significant (HR: 2.37; 95\% CI: 1.111-5.065, univariate and multivariate analyses $\mathrm{p}=0.026$ ), suggesting that actinin-4 protein expression was an independent prognostic factor for stage II and IIIA adenocarcinoma of the lung (Supplementary Table 3). Analysis of the effect of adjuvant chemotherapy indicated no significant difference 

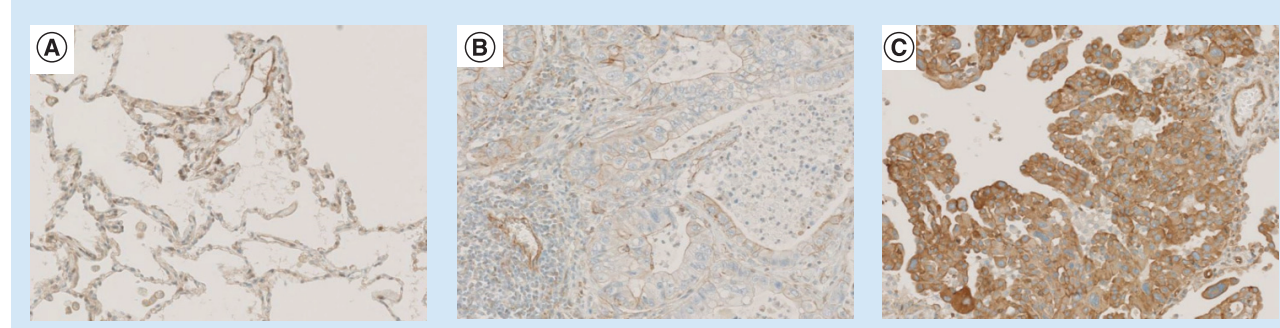

(D)

(E)

All patients
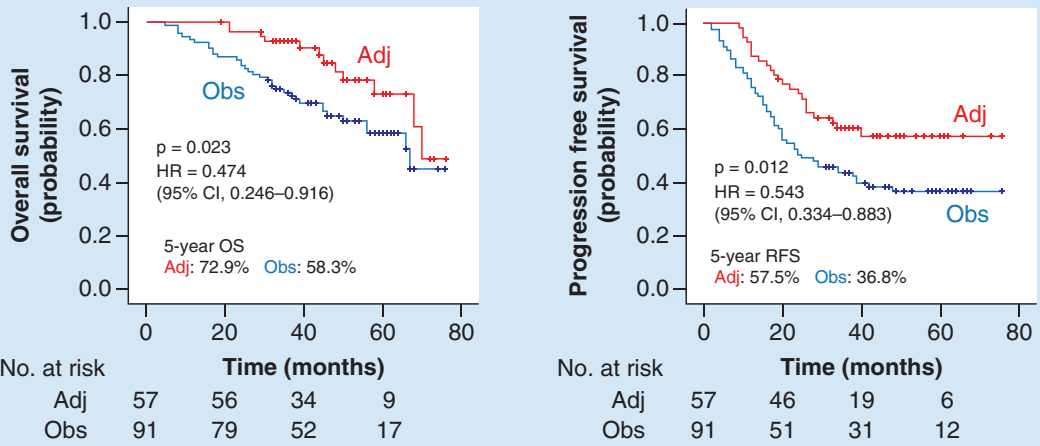

(F)

Actinin-4-IHC(+) group

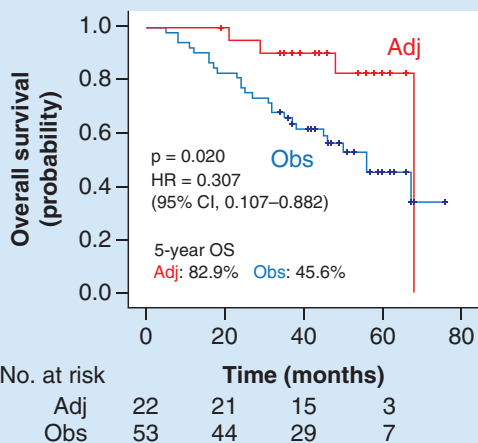

(G)

Actinin-4-IHC(+) group

(H)

Actinin-4-IHC(-) group

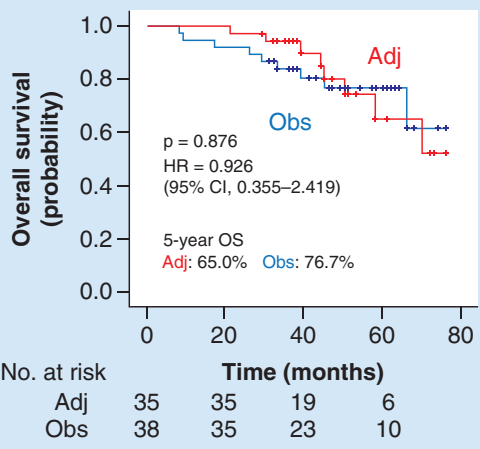

(1)

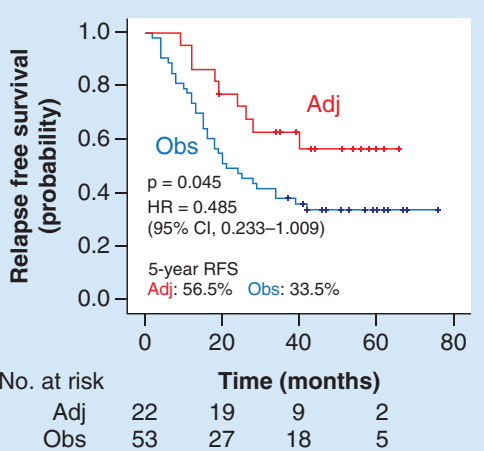

Actinin-4-IHC(-) group

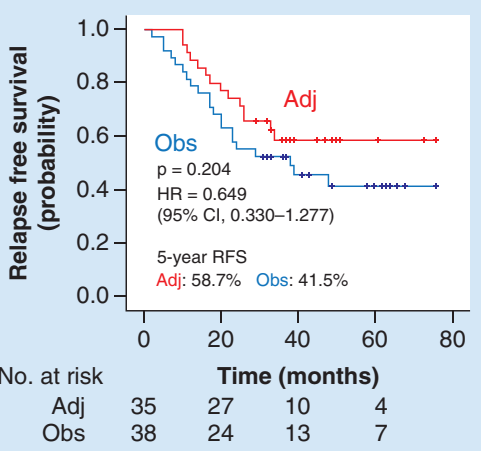

Figure 2. Expression of the actinin-4 protein in adenocarcinoma of the lung, and in the epithelia of alveoli and Kaplan-Meier curves of overall survival and recurrence-free survival in all patients. Representative actinin-4 protein expression in the epithelia of alveoli ( $A$ ) and in adenocarcinoma of the lung (B \& ), as determined using immunohistochemistry (IHC). Tissues negative (B) or positive (C) for actiin-4 protein expression are shown. Kaplan-Meier curves of OS (D) and RFS (E) of patients who underwent adjuvant chemotherapy (Adj: red line) or who were observed without adjuvant chemotherapy (Obs: blue line) are shown. OS (F \& H) and RFS (G \& I) of Kaplan-Meier curves of Actinin-4-IHC(+) (F \& G) or actinin-4-IHC(-) (H \& I) patients who underwent adjuvant chemotherapy (Adj: red line) or who were observed without adjuvant chemotherapy (Obs: blue line) are shown. Statistically significant differences ( $p$-values) between the survival times of Adj and Obs patients were calculated using a log-rank test. Hazard rations (HRs) for the risk of death and $95 \% \mathrm{Cls}$ were calculated using Cox regression univariate analysis. IHC: Immunohistochemistry; OS: Overall survival; RFS: Recurrence-free survival. 


\begin{tabular}{|c|c|c|c|c|c|}
\hline & & \multicolumn{2}{|c|}{ Univariate } & \multicolumn{2}{|c|}{ Multivariate } \\
\hline & & HR (95\% Cl) & $p$-value & HR $(95 \% \mathrm{Cl})$ & p-value \\
\hline Age (years) & $\geq 65$ vs $<65$ & $1.549(0.734-3.269)$ & 0.25 & & \\
\hline Sex & Male vs female & $0.453(0.216-0.948)$ & 0.036 & $0.487(0.232-1.022)$ & 0.057 \\
\hline Smoking status & Ex vs never & $0.716(0.337-1.518)$ & 0.383 & & \\
\hline p-TNM Stage & IIIA vs IIA.IIB & $1.066(0.520-2.186)$ & 0.862 & & \\
\hline $\mathrm{CCl}$ & $\geq 1$ vs 0 & $1.792(0.835-3.846)$ & 0.134 & & \\
\hline Treatment & $\begin{array}{l}\text { Chemotherapy vs } \\
\text { observation }\end{array}$ & $0.307(0.107-0.882)$ & 0.028 & $0.328(0.114-0.945)$ & 0.039 \\
\hline \multicolumn{6}{|c|}{ Cox analyses of potential prognostic factors for OS in the actinin-4 negative group } \\
\hline Age (years) & $\geq 65$ vs $<65$ & $1.542(0.567-4.193)$ & 0.396 & & \\
\hline Sex & Male vs female & $0.620(0.299-1.680)$ & 0.347 & & \\
\hline Smoking status & Ex vs never & $0.647(0.249-1.684)$ & 0.372 & & \\
\hline p-TNM Stage & IIIA vs IIA.IIB & $2.511(0.883-7.145)$ & 0.084 & & \\
\hline $\mathrm{CCl}$ & $\geq 1$ vs 0 & $1.087(0.354-3.342)$ & 0.884 & & \\
\hline Treatment & $\begin{array}{l}\text { Chemotherapy vs } \\
\text { observation }\end{array}$ & $0.926(0.355-2.419)$ & 0.876 & & \\
\hline
\end{tabular}

in OS between actinin-4-IHC( + ) and actinin-4-IHC(-) subgroups ( $\mathrm{p}=0.846, \log$-rank test), and the 5-year OS rates were 82.9 and $65.0 \%$, respectively (Supplementary Figure 2).

\section{Prediction of the efficacy of adjuvant chemotherapy by IHC analysis of actinin-4}

On Kaplan-Meier analysis, the Adj group had better OS and RFS than the Obs Group ( $\mathrm{p}=0.023$, log-rank test) and significantly higher 5-year OS and RFS rates (72.9 and 57.5\%, respectively) than the Obs group (58.3 and $36.8 \%$, respectively) (Figure 2D \& E).

On analysis of actinin-4 IHC patterns, the patients in each group were then classified into two subgroups based on actinin-4 protein positive or negative expression (positive and negative subgroups, respectively). Kaplan-Meier analysis of the actinin-4-IHC(+) subgroups indicated that the prognosis of the actinin-4-IHC(+) Adj subgroup was significantly better than that of the actinin-4-IHC( + ) Obs subgroup $(\mathrm{p}=0.028$ log-rank test), and the 5-year OS of the actinin-4-IHC(+) Adj subgroup was better than that of the actinin-4-IHC(+) Obs subgroup (82.9 vs $45.6 \%$ ), and it was improved compared with that of the total Adj group (Figure 2F). In contrast, in the actinin-4-IHC(-) subgroups, there was no improvement in the 5-year OS of the Adj subgroup (65.0\%) compared with that of the Obs subgroup $(76.7 \%)$. Moreover, there was no significant difference between the survival curves of the actinin-4-IHC(-) Adj and Obs groups (Figure 2H).

Regarding RFS, the RFS of the actinin-4-IHC(+) Adj subgroup was significantly longer than that of the actinin4-IHC(+) Obs group ( $\mathrm{p}=0.045$, log-rank test) (Figure 2G). However, there was no significant difference between the RFS curves of the actinin-4-IHC(-) Adj and Obs groups ( $\mathrm{p}=0.204$, log-rank test) (Figure 2I).

The HR of death calculated for all patients without subgroup analysis using the Cox proportional hazard model was significantly lower in the Adj group than in the Obs group (HR: 0.474; 95\% CI: 0.246-0.916, univariate analysis) (Figure 2D). In contrast, in the actinin-4-IHC(+) subgroups, the HR for death in the Adj group was significantly improved compared with the Obs group (HR: 0.307; $\mathrm{p}=0.028$; 95\% CI: 0.107-0.882, Cox proportional univariate analysis) (Table 3). Although sex and the status of adjuvant chemotherapy remained significant on univariate analysis of the actinin-4-IHC(+) subgroups, the adjuvant chemotherapy status was identified as the most significant independent prognostic factor on Cox proportional multivariate analysis (HR: 0.328; 95\% CI: 0.114-0.945; $\mathrm{p}=0.039)$. The HR of the actinin-4-IHC $(+)$ subgroups for death $(0.307)$ was lower than that for all patients that were not selected by actinin-4 (0.474). In contrast, in the actinin-4-IHC(-) subgroups, Cox proportional univariate analysis did not indicate any improvement in HR by adjuvant chemotherapy (HR: 0.926; 95\% CI: 0.355-2.419; $\mathrm{p}=0.876$ ) (Table 3). Significant differences were also not recognized for other clinical factors, such as age, sex, smoking status, p-stage and CCI. 


\section{Discussion}

In this retrospective study, actinin-4 was identified as a novel candidate biomarker for prediction of the efficacy of adjuvant chemotherapy with platinum-combination therapy in stage II-IIIA adenocarcinoma of the lung.

Actinin- 4 was investigated as a potential biomarker for the metastatic potential of cancer cells in this study because actinin- 4 functions in the production of cellular protrusions that are involved in cancer invasion [16,17,23,24]. Thus, actinin- 4 overexpression confers metastatic potential on cancer cells and is closely associated with cancer invasion and metastasis in some kinds of cancers. We had also identified actinin- 4 as a prognostic biomarker for invasive ductal breast cancer [15]. Moreover, amplification of the ACTN4 gene, which is one cause of actinin-4 protein overexpression, was identified in pancreatic cancer [23], ovarian cancer [18], salivary gland carcinoma [17] and adenocarcinoma of the lung [22].

In patients that were observed without adjuvant chemotherapy, OS of the actinin-4-IHC (+) subgroup was significantly shorter than that of the actinin-4-IHC(-) subgroup, as was seen for gene amplification of ACTN4 in stage I adenocarcinoma of the lung. In contrast, no significant difference in OS was found between the actinin-4$\mathrm{IHC}(+)$ and actinin-4-IHC(-) subgroups in patients undergoing adjuvant chemotherapy. These data suggest that protein expression of actinin- 4 may not be a simple prognostic biomarker.

Although survival in patients with and without adjuvant chemotherapy could not be directly compared because of selection bias, the present data suggested that actinin-4 protein expression might be not only a prognostic biomarker for patients who are observed without adjuvant chemotherapy but also a predictive biomarker for the efficacy of adjuvant chemotherapy in stage II and IIIA adenocarcinoma of the lung. In addition, the HR for death in the adjuvant chemotherapy actinin-4-IHC(+) subgroup compared with the nonadjuvant therapy actinin-4-IHC(+) subgroup was lower than the HR for death of the adjuvant chemotherapy in all patients (HR: 0.474; 95\% CI: 0.246-0.916, Cox regression univariate analysis) (Figure 2D \& F \& Table 3A \& B). It was therefore considered that IHC analysis of tumor specimens with an anti-actinin- 4 antibody might be useful for personalized treatment of adenocarcinoma of the lung by identifying patients with stage II or IIIA adenocarcinoma who have the potential to benefit from adjuvant chemotherapy with a platinum combination. On the other hand, there was no meaningful association of recurrence pattern with actinin-4-IHC status (Supplementary Table 2A).

We recently demonstrated the possibility that overexpression of ACTN4 mRNA in patients with stage IB and II NSCLC can predict the efficacy of adjuvant chemotherapy with vinorelbine and cisplatin [25]. Our finding was based on re-analysis of a database constructed by JBR.10, which was a prospective study to prove the clinical benefit of adjuvant chemotherapy with vinorelbine and cisplatin for NSCLC. Re-analysis of the comprehensively enrolled mRNA expression profiles that were analyzed by JBR.10 indicated that the ACTN4 mRNA overexpression subgroup received significant clinical benefit from adjuvant chemotherapy with vinorelbine and cisplatin (HR: 0.273; 95\% CI: 0.079-0.952). In contrast, ACTN4 mRNA weak or negative expression subgroups did not receive clinical benefit from adjuvant chemotherapy (HR: 1.008; 95\% CI: 0.574-1.765).

Moreover, we established a cell line of adenocarcinoma of the lung in which actinin-4 protein expression was reduced by siRNA. Although this cell line was not sensitive to cisplatin, interestingly its metastatic ability was significantly reduced [25].

These data suggest that the tumors of patients who overexpress the actinin- 4 protein have metastatic ability without a decrease in chemosensitivity to cisplatin, and that such patients with lung adenocarcinoma should receive platinum-based adjuvant chemotherapy after surgery. Our previous study suggested that protein overexpression and gene amplification of ACTN4 were prognostic biomarkers for patients with lung adenocarcinoma who never underwent adjuvant chemotherapy. In contrast, in this present study, ACTN4 appears to be a potential biomarker for the efficacy of adjuvant chemotherapy for stage II and III NSCLC. Because patients with overexpression of ACTN4 have higher metastatic ability than patients without overexpression of ACTN4, the minimal residual diseases that could not be removed by surgical treatment could be controlled by adjuvant chemotherapy.

One of the limitations of the present study is that it had a small sample size and was a retrospective study. This study had a selection and observation bias because the attending physician decided whether the patient was treated with adjuvant chemotherapy depending on PS, age, concomitant disease and patient preference. To validate the clinical usefulness of a biomarker, it is best to validate it in another cohort that was independently collected. Since the Japanese clinical guideline has recommended adjuvant chemotherapy for patients with stage II and IIIA NSCLC, it was difficult to collect enough recent samples of stage II and III NSCLC patients who never underwent adjuvant chemotherapy. In order to avoid clinical bias and increase reliability for biomarker identification, the 
samples were retrospectively enrolled according to REMARK, which was advocated by a working group of the National Cancer Institute-European Organization for Research and Treatment of Cancer (NCI-EORTC) as a major recommendation to analyze prognostic significance. We are planning to begin a multicenter, prospective, observational study to examine this marker's possibilities. Ideally, a prospective, randomized study should be conducted to clarify the benefits of tailored adjuvant chemotherapy for patients with stage I lung adenocarcinoma after stratifying them on the basis of actinin- 4 expression.

A second limitation is that the usefulness of this predictive biomarker for NSCLCs including squamous cell carcinoma and large cell carcinoma was not examined. In our previous analysis, Noro et al. reported that patients with adenocarcinoma of the lung with gene amplification of ACTN4 and without adjuvant chemotherapy had a much worse outcome than patients without ACTN4 gene amplification [22]. In particular, the HR for death of the former patients was 10.53 (Cox regression univariate analysis, 95\% CI: 4.15-26.7), and it was higher than the HR between stages IA and IB (HR: 6.89, 95\% CI 2.26-21.0). Thus, ACTN4 gene amplification might be useful as a predictive biomarker for personalized treatment to decide the strategy for adjuvant chemotherapy in stage I adenocarcinoma of the lung. More recently, a prognostic classifier for stage I and II lung squamous cell carcinoma was reported by an international collaborative study. This prognostic classifier consists of a two-gene signature that was measured by comprehensive analysis of mRNA expression. This two-gene signature includes ACTN4. This report demonstrated that overexpression of ACTN4 mRNA is a significant indicator of poor prognosis in stage I and II squamous cell carcinoma of the lung. These data suggested that overexpression of ACTN4 might be a biomarker for prediction of the efficacy of adjuvant chemotherapy not only for adenocarcinoma but also for squamous cell carcinoma of the lung [26]. To confirm this hypothesis, a prospective clinical validation study of a companion biomarker with actinin- 4 for adjuvant chemotherapy including stage I should be designed in the future.

\section{Conclusion}

This retrospective study showed that actinin- 4 protein overexpression assessed by IHC has the potential to be a predictive biomarker of the efficacy of adjuvant chemotherapy for patients with stage II or IIIA adenocarcinoma of the lung. This biomarker could help identify patients who would derive the greatest benefit from adjuvant chemotherapy.

\section{Financial \& competing interests disclosure}

This work was supported by the Japan Agency for Medical Research and Development (AMED) Practical Research for Innovation Cancer Control (15ck0106101h0002, K Honda), the AMED Project for Cancer Research And Therapeutic Evolution (P-CREATE) (17cm0106403h0002, K Honda), AMED CREST (17gm0710010h0104 and 17gm0710013h0304, K Honda), a Grant-in Aid for Scientific Research (B) (16H05547), and by the Ministry of Education, Culture, Sports, Science and Technology (METX) of Japan $(16 \mathrm{~K} 15833, \mathrm{~K} \mathrm{H}$.). The authors have no other relevant affiliations or financial involvement with any organization or entity with a financial interest in or financial conflict with the subject matter or materials discussed in the manuscript apart from those disclosed.

No writing assistance was utilized in the production of this manuscript.

\section{Ethical conduct of research}

This study was conducted with the approval of the Institutional Review Board of the NCC. The procedures followed were in accordance with the ethical standards of the responsible committee on human experimentation (institutional or regional) and with the Helsinki Declaration of the World Medical Association.

\section{Open access}

This work is licensed under the Attribution-NonCommercial-NoDerivatives 4.0 Unported License. To view a copy of this license, visit http://creativecommons.org/licenses/by-nc-nd/4.0/

Supplementary Data

To view the supplementary data that accompany this paper please visit the journal website at www.futuremedicine.com/doi/full/10.2217/bmm-2017-0150 


\section{Summary points}

- Although several clinical trials demonstrated the benefits of platinum-combination adjuvant chemotherapy for stage II and IIIA lung adenocarcinoma, predictive biomarkers for the efficacy of such therapy have not yet been identified.

- We previously showed that protein overexpression of actinin-4 was associated with prognosis in early stage lung adenocarcinoma.

- We measured actinin-4 protein levels in patients with completely resected stage II or IIIA lung adenocarcinoma using immunohistochemistry (IHC) and then retrospectively compared survival between adjuvant chemotherapy and observation groups.

- A total of 148 eligible patients were classified into actinin-4 positive $(n=75)$ or actinin-4 negative cases $(n=73)$ by IHC.

- In the actinin-4 positive cases, patients with adjuvant chemotherapy survived longer than those with observation (HR: 0.307; $p=0.028$ ).

- In contrast, in the actinin-4 negative cases, no significant survival benefit was noted with adjuvant chemotherapy $(H R=0.926 ; p=0.876)$.

- One of the limitations is that this was a small sample size, retrospective study.

- Another limitation is that we did not identify the usefulness of this predictive biomarker for NSCLCs including squamous cell carcinoma and large cell carcinoma.

- Overexpression of actinin-4 assessed by IHC is not only a prognostic marker but also potentially a predictive biomarker of the efficacy of adjuvant chemotherapy for patients with stage II or IIIA adenocarcinoma of the lung.

- We are planning a multicenter, prospective, observational study to examine this possibility.

\section{References}

1 Goldstraw P, Crowley J, Chansky K et al. The IASLC Lung Cancer Staging Project: proposals for the revision of the TNM stage groupings in the forthcoming (seventh) edition of the TNM Classification of malignant tumours. J. Thorac. Oncol. 2(8), 706-714 (2007).

2 Lyons G, Quadrelli S, Jordan P, Colt H, Chimondeguy D. Clinical impact of the use of the revised International Association for the Study of Lung Cancer staging system to operable non-small-cell lung cancers. Lung Cancer 74(2), 244-247 (2011).

3 Arriagada R, Bergman B, Dunant A et al. Cisplatin-based adjuvant chemotherapy in patients with completely resected non-small-cell lung cancer. N. Engl. J. Med. 350(4), 351-360 (2004).

4 Winton T, Livingston R, Johnson D et al. Vinorelbine plus cisplatin vs. observation in resected non-small-cell lung cancer. N. Engl. J. Med. 352(25), 2589-2597 (2005).

5 Douillard JY, Rosell R, De Lena M et al. Adjuvant vinorelbine plus cisplatin versus observation in patients with completely resected stage IB-IIIA non-small-cell lung cancer (Adjuvant Navelbine International Trialist Association [ANITA]): a randomised controlled trial. Lancet Oncol. 7(9), 719-727 (2006).

6 Butts CA, Ding K, Seymour L et al. Randomized Phase III trial of vinorelbine plus cisplatin compared with observation in completely resected stage IB and II non-small-cell lung cancer: updated survival analysis of JBR-10. J. Clin. Oncol. 28(1), 29-34 (2010).

7 Pisters KM, Evans WK, Azzoli CG et al. Cancer Care Ontario and American Society of Clinical Oncology adjuvant chemotherapy and adjuvant radiation therapy for stages I-IIIA resectable non small-cell lung cancer guideline. J. Clin. Oncol. 25(34), 5506-5518 (2007).

8 Pignon JP, Tribodet H, Scagliotti GV et al. Lung adjuvant cisplatin evaluation: a pooled analysis by the LACE Collaborative Group. J. Clin. Oncol. 26(21), 3552-3559 (2008).

9 Beer DG, Kardia SL, Huang CC et al. Gene-expression profiles predict survival of patients with lung adenocarcinoma. Nat. Med. 8(8), 816-824 (2002).

10 Bianchi F, Nuciforo P, Vecchi M et al. Survival prediction of stage I lung adenocarcinomas by expression of 10 genes. J.Clin. Invest. 117(11), 3436-3444 (2007).

11 Chen HY, Yu SL, Chen CH et al. A five-gene signature and clinical outcome in non-small-cell lung cancer. N. Engl. J. Med. 356(1), $11-20$ (2007).

12 Zhu CQ, Ding K, Strumpf D et al. Prognostic and predictive gene signature for adjuvant chemotherapy in resected non-small-cell lung cancer. J. Clin. Oncol. 28(29), 4417-4424 (2010).

13 Lau SK, Boutros PC, Pintilie M et al. Three-gene prognostic classifier for early-stage non-small-cell lung cancer. J. Clin. Oncol. 25(35), 5562-5569 (2007).

14 Potti A, Mukherjee S, Petersen R et al. A genomic strategy to refine prognosis in early-stage non-small-cell lung cancer. N. Engl. J. Med. 355(6), 570-580 (2006). 
15 Honda K, Yamada T, Endo R et al. Actinin-4, a novel actin-bundling protein associated with cell motility and cancer invasion. J. Cell. Biol. 140(6), 1383-1393 (1998).

16 Hayashida Y, Honda K, Idogawa M et al. E-cadherin regulates the association between beta-catenin and actinin-4. Cancer Res. 65(19), 8836-8845 (2005).

17 Watabe $\mathrm{Y}$, Mori T, Yoshimoto $\mathrm{S}$ et al. Copy number increase of ACTN4 is a prognostic indicator in salivary gland carcinoma. Cancer Med. 3(3), 613-622 (2014).

18 Yamamoto S, Tsuda H, Honda K et al. Actinin-4 expression in ovarian cancer: a novel prognostic indicator independent of clinical stage and histological type. Mod. Pathol. 20(12), 1278-1285 (2007).

19 Honda K, Yamada T, Hayashida Y et al. Actinin-4 increases cell motility and promotes lymph node metastasis of colorectal cancer. Gastroenterology 128(1), 51-62 (2005).

20 Greene FL, Sobin LH. A worldwide approach to the TNM staging system: collaborative efforts of the AJCC and UICC. J. Surg. Oncol. 99(5), 269-272 (2009).

21 Mcshane LM, Altman DG, Sauerbrei W et al. Reporting recommendations for tumor marker prognostic studies (REMARK). J. Natl Cancer Inst. 97(16), 1180-1184 (2005).

22 Noro R, Honda K, Tsuta K et al. Distinct outcome of stage I lung adenocarcinoma with ACTN4 cell motility gene amplification. Ann. Oncol. 24(10), 2594-2600 (2013).

23 Kikuchi S, Honda K, Tsuda $\mathrm{H}$ et al. Expression and gene amplification of actinin-4 in invasive ductal carcinoma of the pancreas. Clin. Cancer Res. 14(17), 5348-5356 (2008).

24 Wang MC, Chang YH, Wu CC et al. Alpha-actinin 4 is associated with cancer cell motility and is a potential biomarker in non-small cell lung cancer. J. Thorac. Oncol. 10(2), 286-301 (2015).

25 Miura N, Kamita M, Kakuya T et al. Efficacy of adjuvant chemotherapy for non-small cell lung cancer assessed by metastatic potential associated with ACTN4. Oncotarget 7(22), 33165-33178 (2016).

26 Noro R, Ishigame $\mathrm{T}$, Walsh $\mathrm{N}$ et al. A two-gene prognostic classifier for early-stage lung squamous cell carcinoma in multiple large-scale and geographically diverse cohorts. J. Thorac. Oncol. 12(1), 65-76 (2016). 
